【国際セッション1】

\title{
Possible Mechanism of Inability to Depress Soleus H-reflex during a Standing Position for Elderly Subjects
}

\author{
Masaaki Tsuruike ${ }^{1)}$, Koichi Kitano ${ }^{2)}$ and David Koceja ${ }^{2)}$ \\ ${ }^{1)}$ Health Science Lab. Graduate School of Sport and Exercise Science, Osaka University of Health \\ and Sport Sciences, ${ }^{2}$ Motor Control Lab. Dept Kinesiology and Program in Neuroscience, Indiana \\ University, Bloomington, USA
}

Modulation of the H-reflex has been shown to be different with increasing age. Elderly subjects may not depress the soleus $\mathrm{H}$-reflex from a prone to standing position, although the background EMG activity of the test muscle is increased during the standing position. The purpose of this study was to examine the modulation of the conditioned soleus $\mathrm{H}$-reflex between a young and elderly group during different body weight-bearing orientations. 【Methods】 Fifteen elderly subjects (mean: 67.7, SD: 4.1) and 15 young subjects (mean: 22.8, SD: 2.5) were tested. Subjects were tested under two experimental conditioning test paradigms: 1) paired tibial nerve conditioning to measure homosynaptic depression (HD); 2) femoral nerve conditioning to measure heteronymous Ia facilitation $(\mathrm{HF})$. Each conditioning test protocol was evoked and measured on the soleus $\mathrm{H}^{-}$ reflexes during six different postural orientations: 1) supine; 2) supine inclined by 30 degrees of back support; 3) standing with 50\% of body weight-bearing orientation; 4) standing with $75 \%$ of body weight-bearing orientation; 5) standing with 100\% of body weight-bearing orientation; and 6) standing with $125 \%$ of body weight-bearing orientation. A 2 × 6 repeated measures ANOVA was used to examine H/M-max. A $2 \times 3 \times 6$ repeated measures ANOVA was also used to measure the conditioning test protocol. 【Results】 The elderly significantly increased the soleus background EMG during 100\% and 125\% body weight-bearing orientations when compared with the supine position. The elderly significantly increased the H/M-max during standing with $50 \%$ body weightbearing orientation, compared with the back support positions, which was also to impose $50 \%$ of the body weight on the both legs. In contrast, the young significantly decreased the H/M-max from the back support position to the standing with $50 \%$ body weight-bearing orientation. The young significantly modulated the $\mathrm{HF}-\mathrm{H}$-reflexes across the different body weight-bearing orientations, whereas the elderly did not. The elderly significantly decreased HD-H-reflex during 125\% body weight-bearing orientation when compared with that of the supine position. In other words, the elderly increased the amplitude of the test H-reflex, whereas the young did not. 【Conclusion】 The 50\% body weight-bearing was equally adjusted. The one was the back support inclined by 30 deg; and the other one was $50 \%$ of the body weight reduction by vertically lifting with the harness. The elderly might not utilize the input from the vestibulospinal tract which activated the interneurons of the first-order primary afferent depolarization. The elderly might develop independent of the ongoing presynaptic inhibition because of the no significant modulation of $\mathrm{HF}-\mathrm{H}-\mathrm{reflex}$. The elderly further enhanced the net excitability of the soleus motoneuron pool as they increased the body-weight bearing, which led to a decrease in the HD-H-reflex. Taken together, the elderly increased the H/M-max during the standing, as compared with non-weight bearing orientation. References: Hultborn, et al. (1987). J Physiol, 389:757-772. Pierrot-Deseilligny. (2002). Muscle \& Nerve, 26(2):67-80. Rudomin, \& Schmidt. (1999). Exp Brain Res, 129 (1):1-37. 